\title{
Never cross the path of a traveling salesman: The neural network generation of Halstead-Reitan trail making tests
}

\author{
DOUGLAS VICKERS and MICHAEL D. LEE \\ University of Adelaide, Adelaide, South Australia, Australia
}

\begin{abstract}
The Halstead-Reitan Trail Making Test (TMT) is one of the most widely used neuropsychological instruments for the assessment of brain damage. Despite its usefulness, however, the TMT has two major disadvantages. It has not been constructed in a principled manner that would facilitate systematic investigation, and there is no established procedure for generating equivalent, but stochastically different, test forms. The reason is that the generation of self-avoiding TMT pathways resembles the finding of near-optimal solutions to the Euclidean Traveling Salesman Problem (TSP) and constitutes a computational problem that is NP-complete. This article describes a practical approach to the problem of generating stochastically different test forms. This approach employs an elastic net neural network to generate TMT forms based on self-avoiding, near-optimal paths, and closed circuits. The usefulness and limitations of this solution are discussed briefly in relation to alternative and complementary problems and procedures.
\end{abstract}

For over half a century, various forms of visually guided path-finding and path-following tasks have been used in experimental and, particularly, in clinical work with human subjects. Of such tests, the most widely used is the Trail Making Test (TMT), which was initially included as a performance subtest in the Army Individual Test of General Ability and was later added to the HalsteadReitan Neuropsychological Battery (Reitan, 1955, 1958, 1992; Reitan \& Wolfson, 1988, 1992).

As routinely administered, the TMT consists of two parts. In Part A, which is shown in Figure 1, 25 numbered circles are "randomly distributed" on a page. The subject's task is to connect the circles, as quickly as possible, by drawing a continuous line, or "trail," from the circle numbered 1 up to that numbered 25 . Part B is similar, except that each of the 25 circles contains a number or a letter, and the circles must be connected in the order $1, \mathrm{~A}, 2, \mathrm{~B}, \ldots, \mathrm{L}, 13$. Performance is monitored by the experimenter or clinician, and errors must be corrected before continuing. For both Parts A and B, the score is the total time needed to complete the task correctly.

The TMT is reputedly one of the most sensitive indicators of brain damage (Corrigan \& Hinkeldey, 1987; Crawford, Parker, \& McKinlay, 1992; Lezak, 1995; Spreen

The present study is part of an ongoing project on the assessment of cognitive functioning carried out at the Psychology Department, University of Adelaide, in collaboration with the Department of Anaesthesia and Intensive Care at the Royal Adelaide Hospital, Adelaide, South Australia. The study was supported by an Adelaide University Research Scheme Grant to D.V. Correspondence concerning this article should be addressed to D. Vickers, Psychology Department, University of Adelaide, Adelaide, South Australia 5005, Australia (e-mail: doug.vickers@ psychology.adelaide.edu.au).
\& Strauss, 1991). In addition, because of its simplicity, low cost, and ease of administration, the TMT is particularly advantageous for clinical applications, and it is perhaps the most frequently employed neuropsychological test ever developed (Butler, Retzlaff, \& Vanderploeg, 1991).

Unfortunately, however, the TMT also suffers from two major disadvantages. The first, and most basic, problem is that the test has not been constructed in accordance with a set of clearly specifiable principles which can be implemented by an algorithm capable of generating other systematically different test forms. As pointed out by Geffen (1995), in a more general critique of approaches to neuropsychological assessment, one consequence of this is that there has been no systematic, experimental study of path-following performance, and little progress has been made in theorizing about an obviously fundamental ability. A second problem is that there is no procedure for generating (theoretically or measurably) equivalent but stochastically different test forms, with the result that very few alternative test forms are available for instruction, experimentation, or repeated testing.

Vickers, Vincent, and Medvedev (1996) have provided a partial remedy for these problems by noting that although the TMT has never been accompanied by an explicit rationale, in practice TMT pathways are markedly different from those which would result if the "randomly distributed" circles had been assigned numbers and letters in a random manner. Specifically, TMT pathways are much shorter and exhibit no self-intersections. It seems likely that paths $\mathrm{A}$ and $\mathrm{B}$ were constructed by first drawing "random-looking" curves and then sequentially assigning the numbers and letters to successive inflection points on these curves. The constraint of self-avoidance, which appears to have been observed in the construction of all 


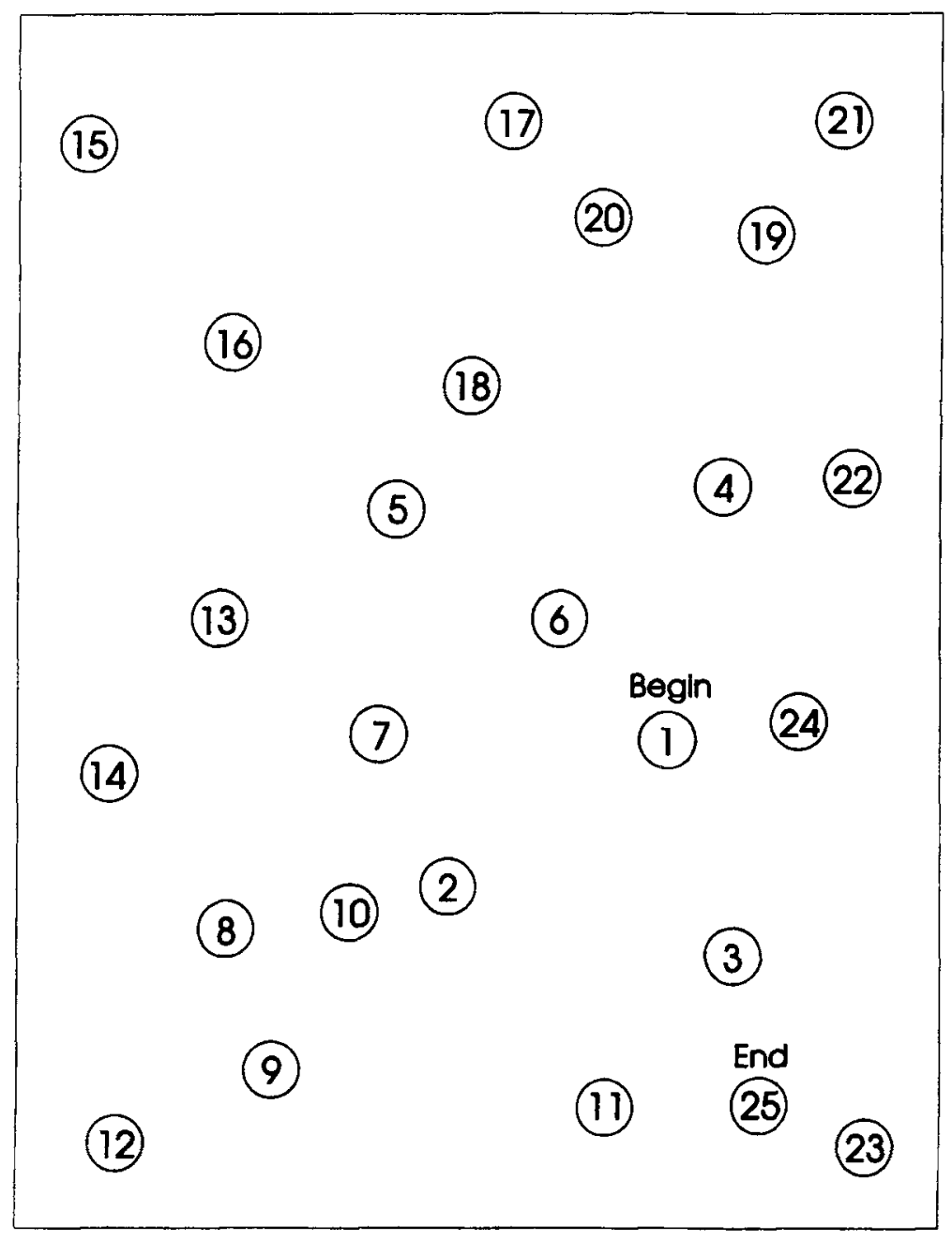

Figure 1. Part A of the TMT.

TMT pathways, was presumably intended to facilitate the task of monitoring and scoring the subject's performance. Such curves, Vickers et al. argue, resemble certain fractal, "space-filling" constructions (Mandelbrot, 1982), and indeed they found that paths A and B had box-covering dimensions of 1.207 and 1.225 , respectively (Voss, 1988). On this basis, Vickers et al. point out that the iterated function systems employed to generate fractals can be directly applied to the construction of systematically varying TMT pathways (Barnsley, 1988a, 1988b; Peitgen, Jürgens, \& Saupe, 1992). Vickers et al. go on to illustrate how the structure of the pathways generated by such systems can be manipulated by varying the complexity and configuration of various elements of the system.

As noted by Lee, Brown, and Vickers (1997), however, the nature of these manipulations makes it difficult to produce with this method large numbers of pathways that are equivalent in complexity but stochastically different. In providing a technique for the principled characterization of TMT pathways in terms of a minimal number of system elements, Vickers et al. effectively place nontrivial constraints on the pathways that their method can generate. Since multiple alternative pathways are required for repeated testing and instruction, the development of a method for generating an unlimited number of stochastically different TMT test forms is highly desirable. The principal purpose of the present paper is to present such a method.

\section{THE TMT AND THE TRAVELING SALESMAN PROBLEM}

Our method hinges on the application of neural networks to problems of combinatorial optimization (see Peterson \& Söderberg, 1995, for an overview), and, in particular, on their application to the notorious Traveling Salesman problem (TSP), a member of the general class of computationally intractable "NP-complete" problems (Goldschlager \& Lister, 1988; Lawler, Lenstra, Rinnooy Kan, \& Shmoys, 1985). The TSP can be characterized as follows: Given a set of $n$ cities, and a specified cost or dis- 


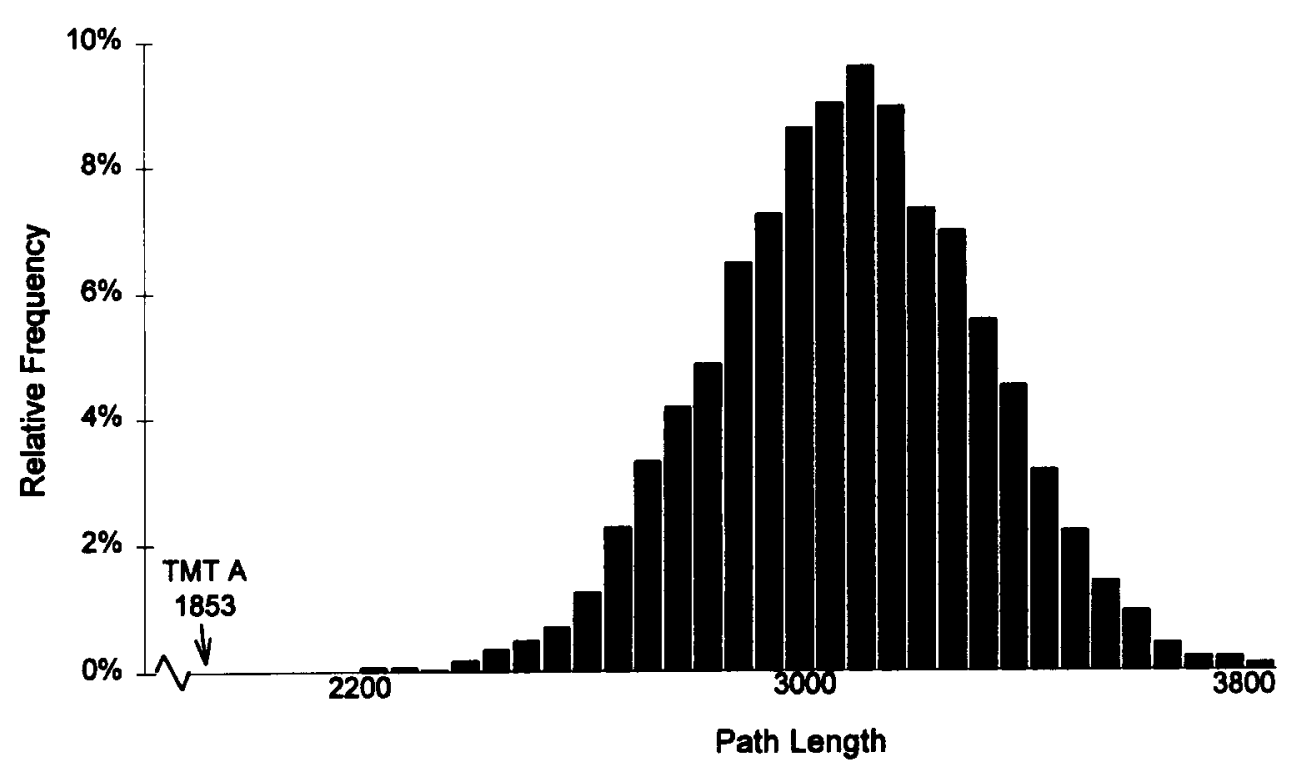

Figure 2. Position of TMT A test form length in the distribution of a sample of 10,000 random paths through the TMT A points.

tance incurred in moving between any two of them, devise an itinerary such that (1) each city is visited exactly once, and (2) the total cost or distance of the itinerary is a minimum.

It seems clear that a direct consequence of the distance minimization requirement is that optimal Euclidean TSP itineraries tend to be self-avoiding, since the crossing of a previously traveled path between two cities generally serves to lengthen the total distance of the journey. Thus, if cities are equated with the circles that define a TMT pathway, an optimal (or even a near-optimal) TSP itinerary is likely to exhibit the self-avoidance that structurally characterizes actual TMT test forms.
Although this insight appears to offer a method for generating alternative TMT test forms, a legitimate initial reservation must be addressed; it may be expressed as follows: If TMT test forms share the structural property of self-avoidance with optimal TSP itineraries, do TMT forms also share the distance minimization properties of TSP solutions? In other words, might the achievement of self-avoidance through adoption of the TSP approach be overly restrictive, in the sense that it generates TMT forms that do not have sufficiently long path lengths?

This concern is substantially addressed by noting that the actual path lengths of both Parts A and B of the TMT are extremely short in comparison with randomly gener-

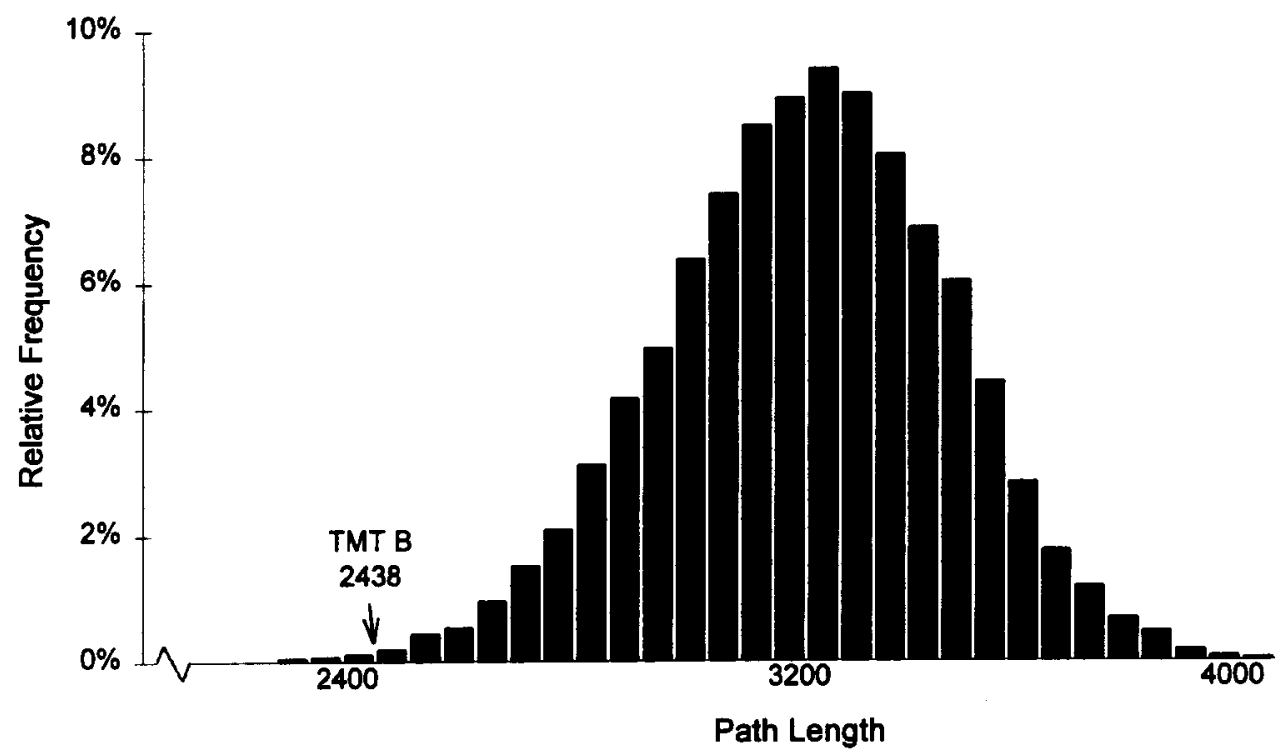

Figure 3. Position of TMT B test form length in the distribution of a sample of 10,000 random paths through the TMT B points. 


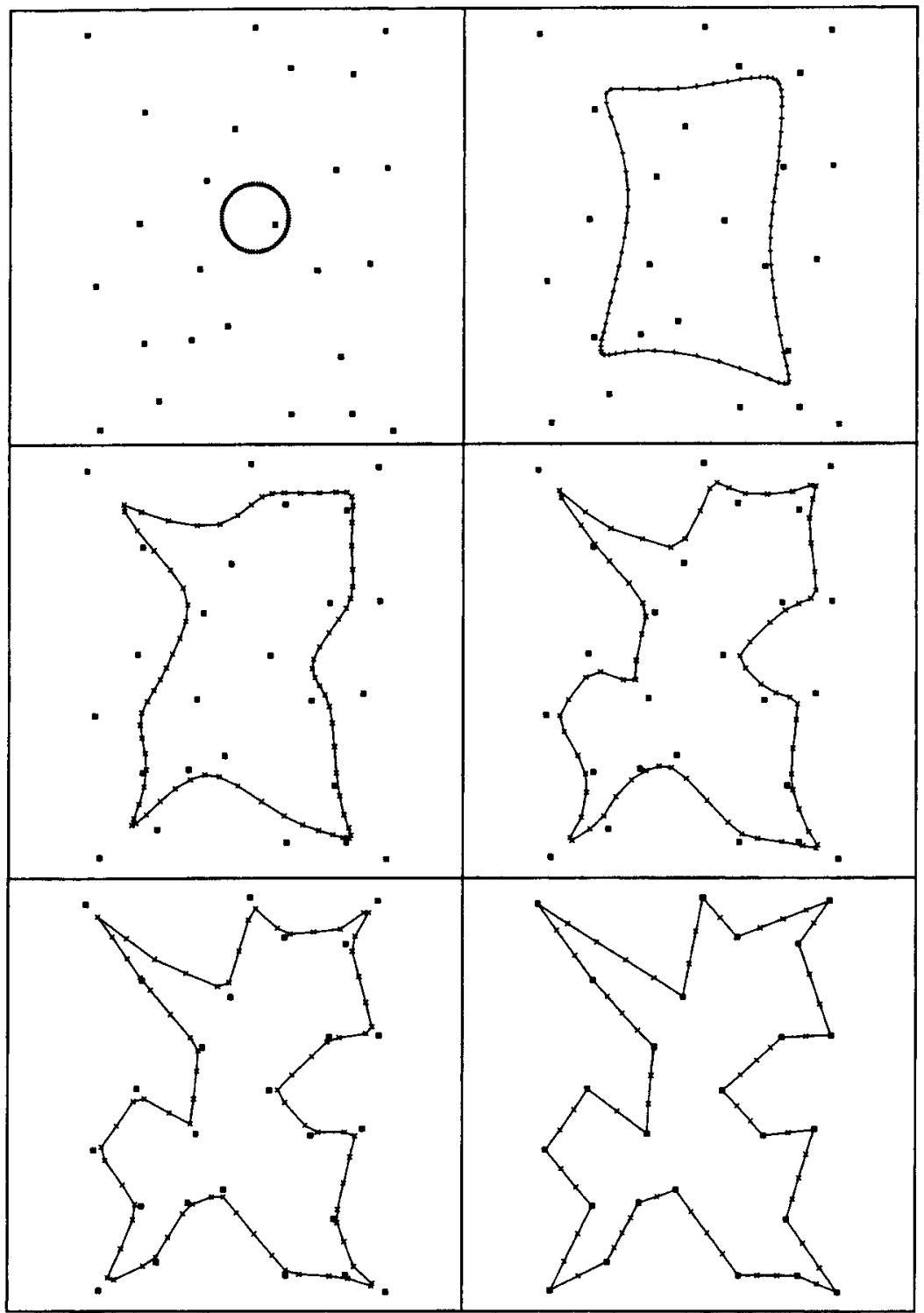

Figure 4. Six stages in the construction of a TSP tour by an elastic net.

ated paths passing through the same set of points. To demonstrate this, Figure 2 depicts the placement of the TMT A path length of $1,853 \mathrm{~mm}$ within the distribution of path lengths of a sample of 10,000 randomly selected paths passing through the points of the TMT A test form. The mean of this sample is $3,064 \mathrm{~mm}$, with a standard deviation of 239. Other 10,000-element random samples, generated by using the same points, were found to have extremely similar means and standard deviations, suggesting that these parameters appropriately characterize the population of path lengths. Thus, we can conclude that the path length of the TMT A test form is of the order of 5 standard deviations shorter than the average path length through these points.
Figure 3 represents a similar analysis of the distribution of path lengths for Part B of the TMT. Again, the sample shown consists of 10,000 random paths, with a mean of $3,222 \mathrm{~mm}$ and a standard deviation of 264 . The actual path length of $2,438 \mathrm{~mm}$ for the TMT B test form is, in this case, approximately 3 standard deviations shorter than this mean path length.

Extensive explorations of random configurations of 25 points suggest that the fact that the TMT path lengths are extremely short in relation to the population of possible path lengths is a robust consequence of the geometry of path construction. In general, it is very difficult to construct self-avoiding paths that are not considerably shorter than the mean path length. Given the unprincipled 
construction of the TMT, and the absence of any theoretical context within which TMT performance might be interpreted and investigated, the primary objective of this paper is to suggest that TMT pathways may be usefully regarded as near-optimal solutions to TS problems.

\section{THE GENERATION OF TMT TOURS AND PATHS}

Looking at the TMT in this way immediately provides many solutions to the problem of generating stochastically varying test forms (Boyd, Pulleyblank, \& Cornuéjols, 1987; Lawler et al., 1985). We employed a neural network approach to the TSP known as the elastic net method (see Yuille, 1995, for an overview). Durbin and Willshaw's
(1987) algorithm was used, because it is simple, robust, well known, and versatile, and it proved capable of generating near-optimal solutions, in reasonable computational time, to the comparatively small problems presented by the TMT. In addition, it can be depicted graphically in such a way as to facilitate an intuitive understanding of the procedure.

Although there has been no systematic, fully representative study of human performance on the TSP, what evidence there is suggests that human beings would also show near-optimal (rather than optimal or severely suboptimal) performance on such problems (MacGregor \& Ormerod, 1996; Polivanova, 1974). The elastic net algorithm had its origins in a theory initially developed to explain the phenomena of retinotopy in the primary visual

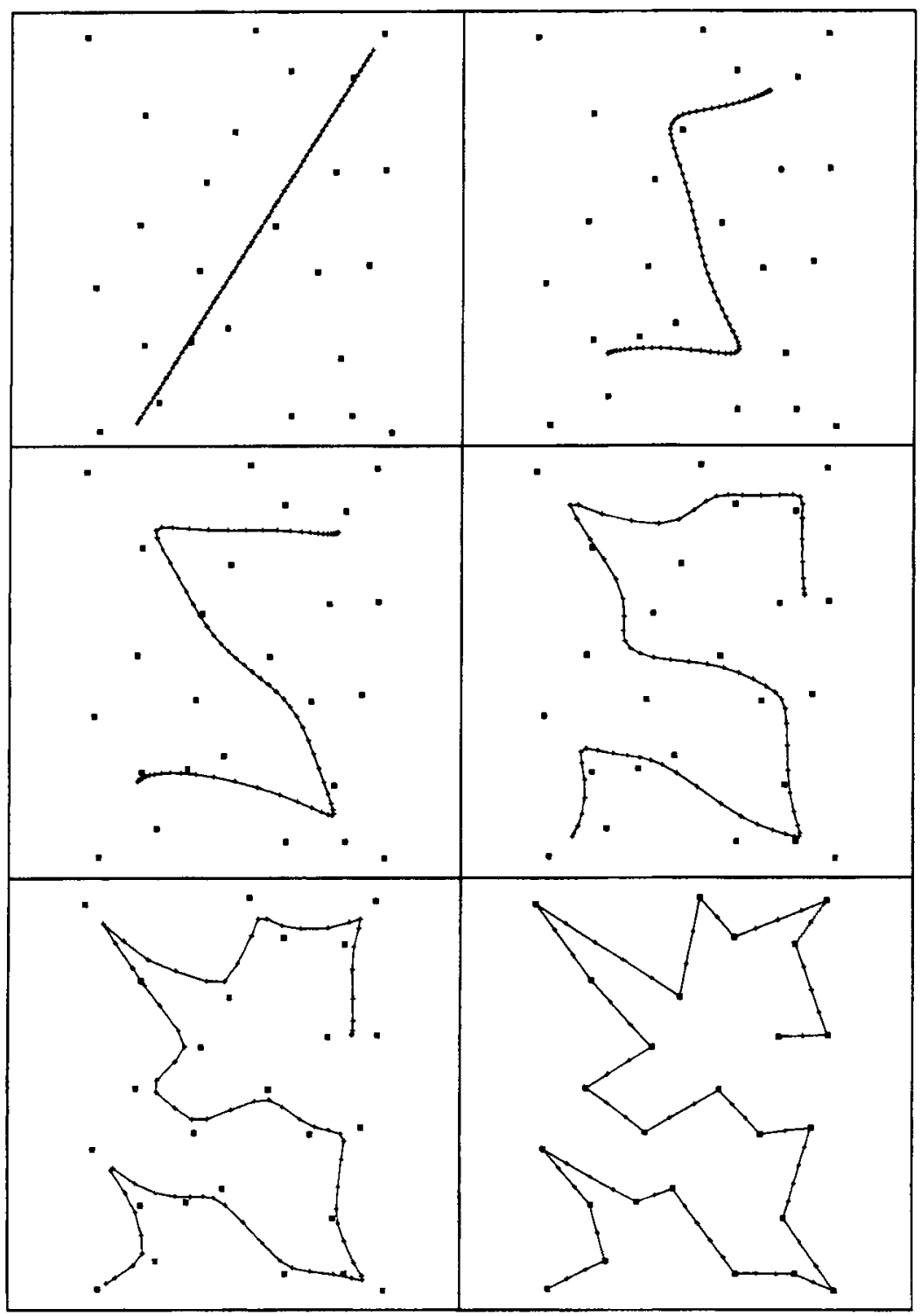

Figure 5. Six stages in the construction of a TSP path by an elastic net, given an initial line structure. 


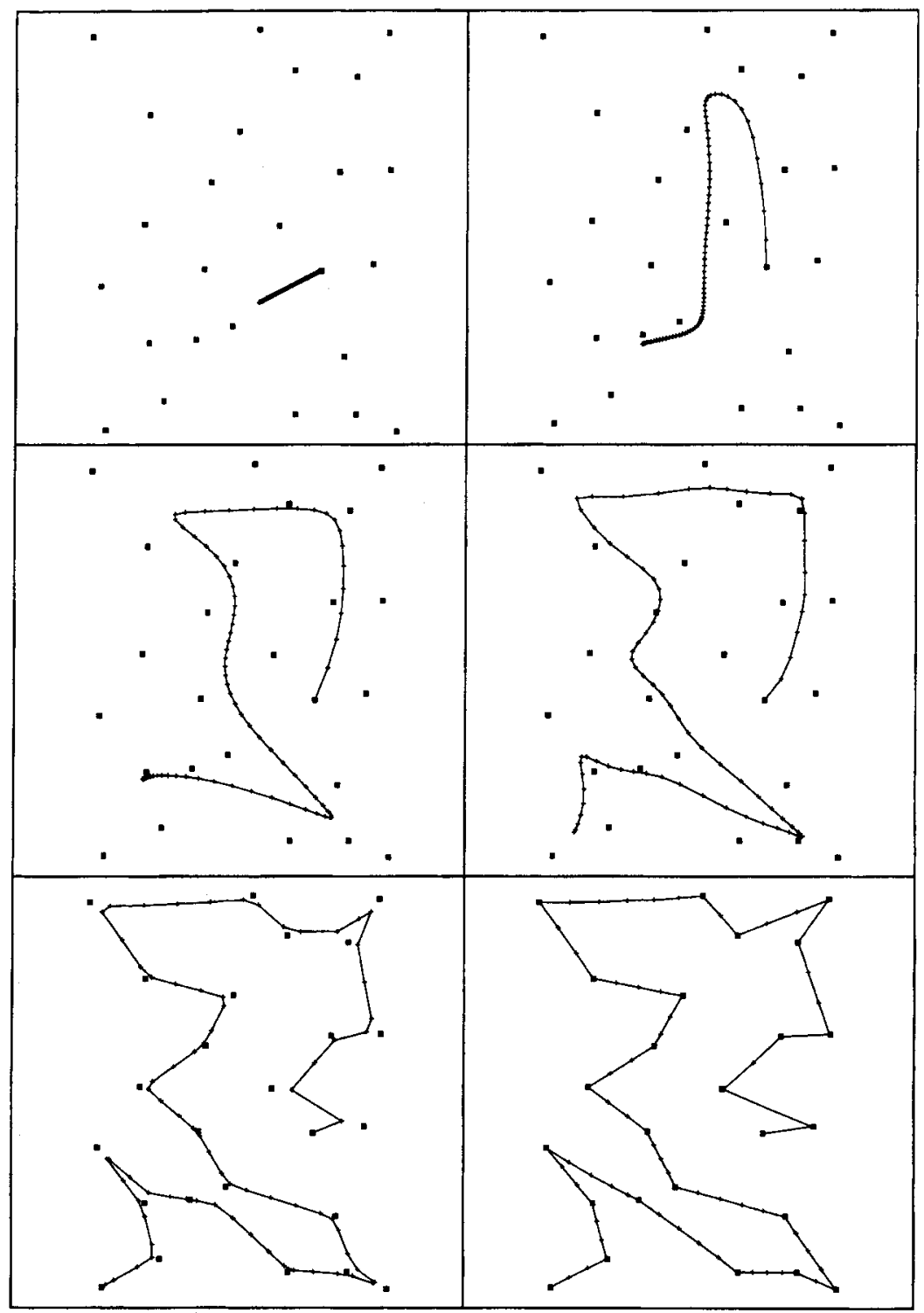

Figure 6. Six stages in the construction of a TSP path by an elastic net, given an initial line structure fixed at one point.

cortex (Malsburg, 1979; Malsburg \& Willshaw, 1977; Willshaw \& Malsburg, 1979). As a parallel, connectionist process, the elastic net would also seem to be a possible candidate as a model of human performance on the TSP. However, it is not necessary that the elastic net constitute a model (plausible or otherwise) of human performance on the TSP or the TMT, any more than that a program for controlling a choice reaction time experiment has to be a model of human decision making. Our primary purpose here is simply to provide a practical method for automatically generating unlimited numbers of alternative TMT forms that are comparable in path complexity to the original forms but that have sufficient stochastic variability to counteract effects of repeated use, practice, and familiarity.
The elastic net method approaches the planar TSPthat is, where the cities are arranged in a plane, so that the cost involved in moving between any two cities corresponds to the (Euclidean) distance between them-by systematically deforming a net to pass through each of the cities. This net is made up of a finite number of elements (typically two or three times greater than the number of cities) which are continually acted on by two forces. The first force seeks to maintain the continuity of the net structure, by keeping each net element near its neighboring net elements. The second force seeks to attract net elements to position themselves on cities, in a such a way that a greater force of attraction exists for the net elements closest to a given city. The cumulative effect of these forces is shown in Figure 4 for a set of 25 randomly lo- 
cated cities, marked as black squares, at four stages during the deformation process. ${ }^{1}$ The elastic net is constructed through the interpolation of 75 net elements, which are also marked.

Elastic nets provide a clear graphical basis on which to understand the relationship between (near) optimal TSP itineraries and TMT pathways. As far as the construction of TMT test forms is concerned, however, they do imply one minor constraint. The nature of the elastic net method results in the starting and finishing points of the derived TMT pathway always being located near each other. That is, the elastic net method essentially generates TSP itineraries that are tours or circuits, starting and finishing at the same point. Although this constraint does not seem to have been deliberately employed in the construction of the original TMT pathways, if the circles numbered 1 and 2 in Part $A$ are renumbered 2 and 1 , respectively, this pathway constitutes a circuit.

Indeed there is some advantage in adding this constraint, since any circle in such touring pathways can be set to be the starting circle. This allows the generation of a far richer set of descriptively equivalent different pathways, beyond those derived from distance preserving transformations (such as rigid rotations), because the sequence of angular changes and segment lengths involved in following the pathways is not invariant across the derived pathways. In addition, there is no obvious reason for thinking that a nontouring pathway structure would

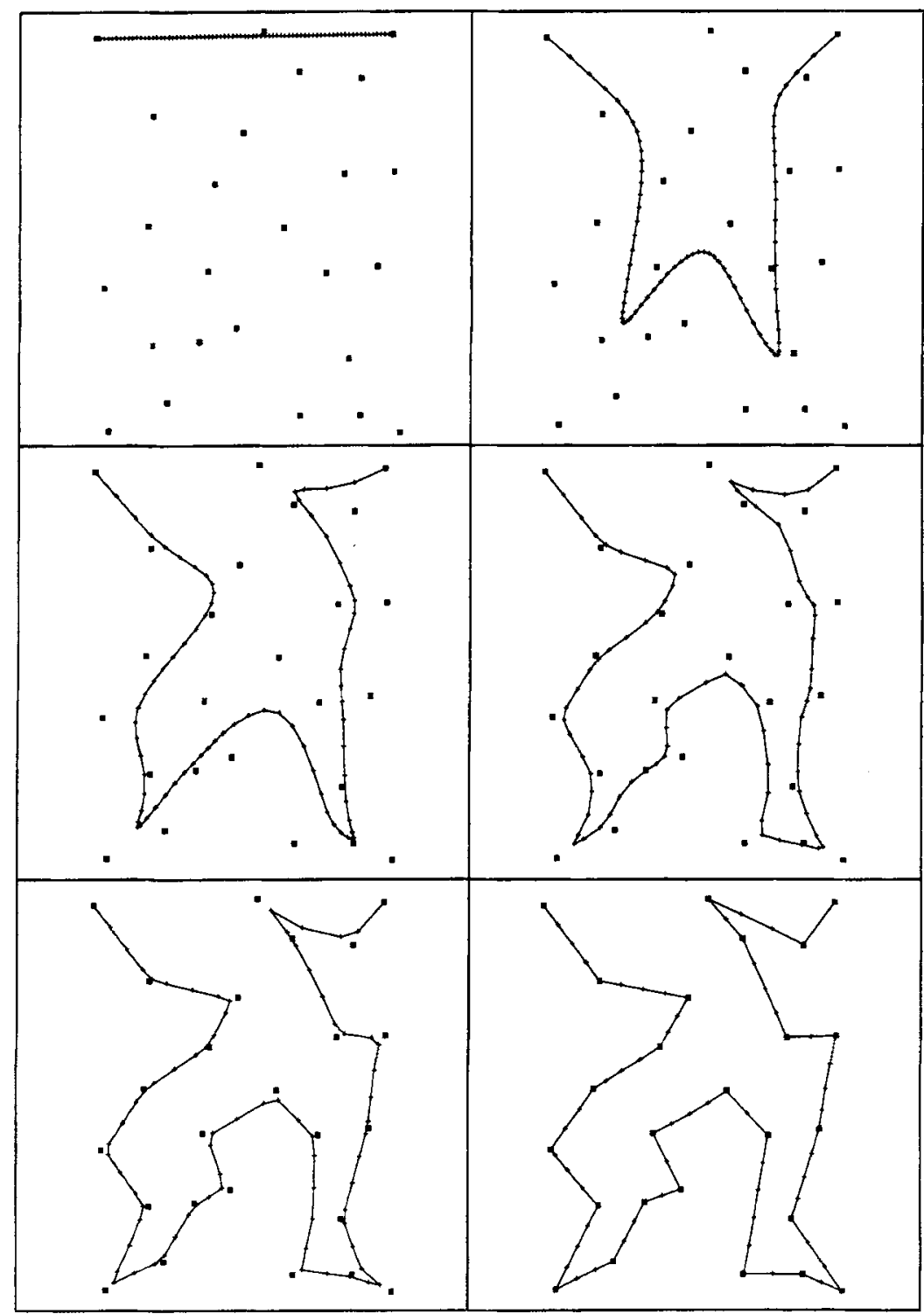

Figure 7. Six stages in the construction of a TSP path by an elastic net, given an initial line structure strung between two points. 
provide a more diagnostic test than one based on a tour: such possibilities remain open to empirical test. In any case, however, a series of modifications to the elastic net approach also allows for the generation of TMT test forms consisting of solution paths other than tours.

The key to generating nontouring paths with elastic nets is to modify the initial circle or ring structure of the net elements. Most simply, if one commences with a line, and removes the association between the first and last elements necessary to maintain the ring structure, the elastic net will construct self-avoiding paths. This process is depicted in Figure 5. Although the algorithm is deterministic, a variety of different paths may be found through the same set of points by utilizing the flexibility available in specifying the endpoints of the initial line location. In our experience, these solutions have path lengths that are several standard deviations below the mean path lengths illustrated in Figures 2 and 3.2

Further variations in the derived path structures may be achieved by assigning a particular starting point for the path, as in Figure 6, or by fixing both starting and finishing points, as in Figure 7.

\section{DISCUSSION}

As one compares these proposed TSP-based methods for generating TMT pathways with the method described by Vickers et al. (1996), it is perhaps useful to consider a continuum that contrasts the principled description of pathways with the constraints placed on their generation. The TSP-based methods that we have presented here represent one extreme on this continuum, in which an arbitrarily placed set of points can form the basis of a TMT pathway, but the method of solution provides no concise description of this pathway. In contrast, the iterated function system method of Vickers et al. generates TMT pathways from a precise and compact foundation, but, so far, the problem of generating stochastic variants of such pathways, which retain the property of self-avoidance, does not seem to have a simple solution.

This distinction can readily be interpreted in terms of the deficiencies of the current form of the TMT described earlier. The first deficiency, regarding the lack of systematic study of path-following performance, is addressed by the iterated function system solution, because it provides the means to relate performance to a small number of pathway-defining characteristics. The second deficiency, regarding the absence of a procedure for generating equivalent but stochastically different test forms, is addressed by the TSP methods, since they allow the generation of a large number of randomly configured pathways, which can then be grouped on the basis of appropriate measures of their complexity.

It should be reiterated that the TSP approach to the construction of path-following tests, outlined above, is limited to the generation of paths for which the internodal distances (between circles or cities) can be repre- sented by a planar arrangement of the nodes in question. Other forms of path-following test exist, such as the "number-joining" or Zahlen-Verbindungs-Test (ZVT), for which this does not hold (Oswald \& Roth, 1987; Vernon, 1993). The original ZVT comprises a $9 \times 10$ matrix of circled numerals from 1 to 90 , which are positioned irregularly, with the sole explicit constraint that successive integers occupy immediately adjacent cells (vertically, horizontally, or diagonally). The subject is required to draw a path through the circled numerals in their natural order, the score being the time required to achieve this. For such matrices, the implied pattern of costs involved in traveling between any two cells cannot be embedded in any metric space. However, other solutions have been developed, involving heuristic approaches (Pearce \& Vickers, 1996), as well as both tree search algorithms and Hopfield-style neural networks, which deal successfully with such problems (Lee et al., 1997).

It could be argued that the TSP approach is also capable of providing a theoretical framework for understanding and investigating path-following performance. The efficiency with which subjects detect and follow optimal (or near-optimal) paths might well be linked to naturally occurring processes of perceptual organization (cf. Pomerantz, 1981). Since many aspects of the life of a biological system can be characterized as problems of optimization (i.e., of maximizing or minimizing the value of some function under multiple constraints), this approach to path following may offer a useful perspective within which to study cognitive efficiency. The consideration of these and other possibilities lies beyond the practical scope of the present paper, but may provide a fruitful avenue for further research.

\section{REFERENCES}

BARNSLEY, M. (1988a). Fractal modelling of real world images. In H.-O. Peitgen \& D. Saupe (Eds.), The science of fractal images (pp. 219 242). New York: Springer-Verlag.

BARNSLEY, M. (1988b). Fractals everywhere. New York: Academic Press. Boyd, S.C., Pulleyblank, W. R., \& CoRnuÉJols, G. (1987). TRAVELan interactive travelling salesman problem package for the IBMpersonal computer. Operations Research Letters, 6, 141-143.

Butler, M., RetzlafF, P. D., \& Vanderploeg, R. (1991). Neuropsychological test usage. Professional Psychology: Theory, Research, \& Practice, 22, 510-512.

Corrigan, J. D., \& Hinkeldey, N. S. (1987). Relationship between Parts A and B of the Trail Making Test. Journal of Clinical Neuropsychology, 43, 402-409.

Crawford, J. R., Parker, D. M., \& McKinlay, W. W. (1992). A handbook of neuropsychological assessment. Hillsdale, NJ: Erlbaum.

Durbin, R., \& Willshaw, D. (1987). An analogue approach to the Traveling Salesman problem using an elastic net method. Nature, 326, $689-691$.

GeFFEN, G. (1995). Approaches to neuropsychological assessment: A comment. Australian Psychologist, 30, 45-46.

GoldSChlager, L., \& Lister, A. (1988). Computer science: A modern introduction (2nd ed.). New York: Prentice-Hall.

Lawler, E. L., Lenstra, J. K., Rinnooy Kan, A. H. G., \& Shmoys, D. B. (1985). The traveling salesman problem: A guided tour of combinatorial optimization. Chichester, U.K.: Wiley.

LEE, M. D., BROWN, M., \& VICKERS, D. (1997). Neural network and tree 
search algorithms for the generation of path-following (trail-making) tests. Journal of Intelligent Systems, 7, 117-143.

LEZAK, M. D. (1995). Neuropsychological assessment (3rd ed.). New York: Oxford University Press.

MACGregor, J. N., \& ORMEROD, T. (1996). Human performance on the traveling salesman problem. Perception \& Psychophysics, 58, 527-539.

MALSBURG, C. VON DER (1979). Development of ocularity domains and growth behavior of axon terminals. Biological Cybernetics, 32, 49-62.

Malsburg, C. von Der, \& Willshaw, D. J. (1977). How to label nerve cells so that they can interconnect in an ordered fashion. Proceedings of the National Academy of Sciences, 74, 5176-5178.

MANDELBROT, B. (1982). The fractal geometry of nature. San Francisco: W. H. Freeman.

OswaLD, W. D., \& Roth, E. (1987). Der Zahlen-Verbindungs-Test (ZVT) Handanweisung (Manual). Göttingen: Hofgrefe.

Pearce, C., \& Vickers, D. (1996). An heuristic for the construction of alternative test forms for the Zahlen-Verbindungs-Test. Perceptual \& Motor Skills, 82, 147-152.

Peitgen, H.-O., Jürgens, H., \& SAuPe, D. (1992). Chaos and fractals: New frontiers of science. New York: Springer-Verlag.

Peterson, C., \& Söderberg, B. (1995). Neural optimization. In M. A. Arbib (Ed.), Handbook of brain theory and neural networks (pp. 617621). Cambridge, MA: MIT Press.

Polivanova, N. I. (1974). [Functional and structural aspects of the visual components of intuition in problem solving]. Voprosy Psikhologii, 4 , 41-51.

Pomerantz, J. R. (1981). Perceptual organization in information processing. In M. Kubovy \& J. R. Pomerantz (Eds.), Perceptual organization (pp. 141-180). Hillsdale, NJ: Erlbaum.

ReITAN, R. M. (1955). The relation of the Trail Making Test to organic brain damage. Journal of Consulting Psychology, 19, 393-394.

REITAN, R. M. (1958). The validity of the Trail Making Test as an indicator of organic brain damage. Perceptual \& Motor Skills, 8, 271-276.

REITAN, R. M. (1992). Trail Making Test: Manual for administration and scoring. Tucson, AZ: Reitan Neuropsychology Laboratory.

REITAN, R. M., \& Wolfson, D. (1988). Traumatic brain injury: Vol. II. Recovery and rehabilitation. Tucson, AZ: Neuropsychology Press.

REITAN, R. M., \& Wolfson, D. (1992). Neuropsychological evaluation of older children. Tucson, AZ: Neuropsychology Press.

SPREEN, O., \& STRAuSs, E. (1991). A compendium of neuropsychological tests. Oxford: Oxford University Press.
Vernon, P. A. (1993). Der Zahlen-Verbindungs-Test and other trailmaking correlates of general intelligence. Personality \& Individual Differences, 14, 35-40.

Vickers, D., Vincent, N., \& Medvedev, A. (1996). The geometric structure, construction, and interpretation of path-following (trailmaking) tests. Journal of Clinical Psychology, 52, 651-661.

Voss, R. F. (1988). Fractals in nature: From characterization to simulation. In H.-O. Peitgen \& D. Saupe (Eds.), The science of fractal images (pp. 21-70). New York: Springer-Verlag.

WILlSHAW, D. J., \& MALSBURG, C. VON DER (1979). A marker induction mechanism for the establishment of ordered neural mappings; its application to the retinotectal problem. Philosophical Transactions of the Royal Society of London: Series B, 287, 203-243.

Yurlle, A. L. (1995). Constrained optimization and the elastic net. In M. A. Arbib (Ed.), Handbook of brain theory and neural networks (pp. 250-255). Cambridge, MA: MIT Press.

\section{NOTES}

1. As was the case with all of the algorithms described here, the elastic net procedure was implemented in Pascal on an IBM-PC-compatible microcomputer.

2. A primary constraint in the construction of TMT paths seems to have been that of self-avoidance, rather than path length. Indeed, TMT paths $A$ and $B$ have significantly different mean segment lengths (Vickers et al., 1996). Nevertheless, we appreciate the desirability of directly comparing the lengths of elastic net solutions and TMT paths. However, the resolution of this issue is much more complicated than simply placing "the elastic net solution" on Figures 2 and 3. Despite the deterministic nature of the elastic net, an infinite number of paths can be generated by altering parameter values and initial conditions. There is, therefore, no single path length that can satisfactorily represent the elastic net approach. Rather, a distribution of lengths would need to be determined. An adequate consideration of this issue is, we believe, an open and difficult research question, which is both outside the aims and beyond the scope of the present paper, particularly in view of the number of free parameters.

(Manuscript received December 19, 1995; revision accepted for publication March 17, 1997.) 